\title{
ESTIMATING SEX-SPECIFIC DISPERSAL RATES WITH AUTOSOMAL MARKERS IN HIERARCHICALLY STRUCTURED POPULATIONS
}

\author{
Pierre Fontanillas, ${ }^{1}$ Eric Petit,${ }^{2}$ And Nicolas Perrin \\ Department of Ecology and Evolution, University of Lausanne, CH-1015 Lausanne, Switzerland \\ ${ }^{1}$ E-mail pierre.fontanillas@ie-zea.unil.ch
}

\begin{abstract}
A recent study suggests that sex-specific dispersal rates can be quantitatively estimated on the basis of sex- and state-specific (pre- vs. postdispersal) $F$-statistics. In the present paper, we extend this approach to account for the hierarchical structure of natural populations, and we validate it through individual-based simulations. The model is applied to an empirical data set consisting of 536 individuals (males, females, and predispersal juveniles) of greater white-toothed shrews (Crocidura russula), sampled according to a hierarchical design and typed for seven autosomal microsatellite loci. From this dataset, dispersal is significantly female biased at the local scale (breedinggroup level), but not at the larger scale (among local populations). We argue that selective pressures on dispersal are likely to depend on the spatial scale considered, and that short-distance dispersal should mainly respond to kin interactions (inbreeding or kin competition avoidance), which exert differential pressure on males and females.
\end{abstract}

Key words._coancestry, Crocidura russula, F-statistics, individual-based models, local competition, variance analysis.

Received July 21, 2003. Accepted December 7, 2003.

Important efforts have recently been devoted to formalize and model the main factors acting on the evolution of dispersal (e.g., Clobert et al. 2001), among which are temporal and spatial heterogeneities in the environment (Hanski 2001; Whitlock 2001; Wiens 2001), inbreeding avoidance (Pusey 1987; Wolff et al. 1988; Pusey and Wolf 1996; Perrin and Mazalov 1999; O' Rian and Braude 2001) and local competition (Perrin and Mazalov 2000; Lambin et al. 2001; Perrin and Goudet 2001). As a result of these efforts, in theory the dispersal patterns expected from the independent action of each of these forces seem by now well appreciated. However, dispersal in practice often results from multiple causes that simultaneously affect behavioral evolution in any given species (Gandon and Michalakis 2001), which might confuse matters when it comes to interpreting dispersal patterns in nature. How can these multiple effects be disentangled in a concrete case study?

One first line consists in contrasting sex-specific patterns. Dispersal is often sex-biased in nature (Greenwood 1980; Dobson 1982; Clarke et al. 1997), and ideas developed to explain dispersal in general can often be tested by their ability to explain differences in dispersal between the sexes (Goudet et al. 2002). Sex biases are most likely to result from kin interactions, be it inbreeding avoidance (Motro 1991, 1994; Gandon 1999; Perrin and Mazalov 1999) or local competition (Perrin and Goudet 2001). Many polygynous mammals, for instance, display a male-biased dispersal, which seems best explained by the strong local mate competition that characterizes their female-defense mating system (Perrin and Mazalov 2000).

A second potentially important source of information on evolutionary causes might be gained from investigations on the distribution of dispersal distances (Murrell et al. 2002; Rousset and Gandon 2002). Long-distance dispersal is unlikely to stem from the same causes as short-distance dis-

\footnotetext{
2 Present address: Ethology, Evolution, and Ecology, UMR 6552, University of Rennes I, Biological Station, F-35380 Paimpont, France.
}

persal (Ronce et al. 2001). Although inbreeding or kin competition might often be avoided by short moves, greater distances are normally required to escape crowding conditions or to colonize vacant territories. Furthermore, dispersal distances, just like dispersal rates, may differ among sexes (Blundell et al. 2002; Yaber and Rabenold 2002). Although short-distance dispersal is expected to display sex biases if kin avoidance plays an important role at a local scale, longdistance dispersal should not if colonization processes constitute the prevailing ultimate cause at this scale. Ji et al. (2001), for example, showed that movement among occupied territories is male biased in the brushtail possum Trichosurus vulpecula, whereas both sexes disperse when it comes to colonize empty sites. Thus, important information on factors acting on the evolution of dispersal can potentially be gained by combining information on sex-specific dispersal rates and distances.

However, before inferring anything about processes, reliable information on patterns must obviously be obtained. Dispersal, unfortunately, seems harder to measure than to model. Direct measurements by mark-and-recapture studies potentially provide the best empirical evidence (Bennetts et al. 2001), and have been used in several instances to document sex-biased rates (e.g., Favre et al. 1997). But markand-recapture studies are spatially and temporally restricted, and therefore reveal only partially the patterns of individual movements. In particular, such studies have often limited ability to reveal long-distance dispersal (Dobson 1994; vanVuren and Armitage 1994; Koenig et al. 1996; Peacock and Ray 2001), unless huge amounts of field work are invested.

These difficulties have prompted the development of indirect approaches, aimed at inferring gene flow from the genetic structure of populations (Slatkin 1985; Rousset 2001). A classical technique consists of assessing $F_{\mathrm{ST}}$ (a measure of genetic differentiation among populations) on the basis of autosomal neutral markers, from which the effective dispersal rate $(\mathrm{Nm})$ can then be calculated using the relation 
$F_{\mathrm{ST}} \approx 1 /(4 N m+1)($ Wright 1943$)$. However, this equation offers only an approximate estimate of gene flow that relies on a series of simplifying assumptions, including infinite island settings and long-term equilibrium dynamics (see Whitlock and McCauley 1999). Moreover, it provides no information on the sex-specificity of dispersal.

In this respect, the use of sex-specific markers (e.g., mtDNA or Y-chromosome), either alone (Petit et al. 2002) or in combination with autosomal markers (Wang 1999; Petit et al. 2001, 2002), provides an a priori promising approach, but has also its limitations. Male-specific markers are not always easy to obtain, and mtDNA evolution might sometimes differ drastically from that of neutral autosomal markers (Rand 1994, 2001). Alternative approaches consist of contrasting the sexspecific distributions of genetic parameters ( $F$-statistics, relatedness values, or assignment indices; Paetkau et al. 1995; Favre et al. 1997; Mossman and Waser 1999; see also review in Prugnolle and de Meeus 2002). These approaches, validated through individual-based simulations (Goudet et al. 2002) have the potential to reveal sex differences in gene flow within a generation, but not to quantify it.

Vitalis (2002) has recently proposed a new method aimed at quantifying sex-specific dispersal rate in an island model on the basis of neutral autosomal markers only. The method relies on a comparison of sex-specific $F_{\mathrm{ST}}$-values evaluated before and after dispersal within a generation. Genetic differentiation among juveniles (sampled before dispersal) is indeed expected to exceed differentiation among adults (either males or females), because dispersal necessarily homogenizes population structure. Differences can thus be used to quantify dispersal in both sexes. This approach, validated through a series of individual-based simulations, appears quite promising, but, in its present form, provides no information on possible differences in dispersal distances.

In the present paper, we propose an extension of Vitalis' (2002) approach that addresses hierarchically structured populations, to contrast sex-specific spatial patterns of dispersal. Hierarchical structures are common in natural populations. Social species, for example, often live in breeding groups (troops, coteries, clans, etc.), which creates an additional level of genetic structure, intermediate between individuals and local populations (colonies). This social structure has strong influences on the dynamics and apportionment of genetic variance, including important effects on inbreeding and effective population sizes (Chesser 1991a, b; Chesser et al. 1993a, b; Sugg et al. 1996; Dobson 1998). Using a simple hierarchical framework (individuals within breeding groups within populations, Slatkin and Voelm 1991; Vigouroux and Couvet 2000), two discrete classes of dispersal distances are to be distinguished. Short-distance dispersal occurs among groups within a local population, and long-distance dispersal occurs among populations. Thus, an analysis of sex-specific $F$-statistics at the several hierarchical levels involved has the potential to provide information on sex-specific differences in both rates and distances of dispersal.

In this paper, (1) we use a coancestry analysis to derive estimators of sex-specific dispersal rate for each of the two hierarchical levels delineated above; (2) we validate this approach through a series of individual-based simulations; and (3) we apply it to an empirical data set consisting of 536 greater white-toothed shrews (Crocidura russula) sampled according to a hierarchical design, and genotyped at seven autosomal microsatellite markers.

\section{THE MODEL}

We consider a metapopulation $(T)$ hierarchically structured into local populations $(S)$, themselves divided into breeding groups $(G)$. The mating system is unimportant, and the life cycle can be annual or perennial with overlapping generations. However, one main assumption is that dispersal occurs at the onset of maturity, so that juveniles are predispersal individuals, whereas adults are postdispersal. Dispersal among populations is written $d_{2}$, and can be sex specific (thus $d_{2 m}$ and $d_{2 f}$ for male and female dispersal, respectively). Similarly, dispersal among breeding groups within populations can be sexspecific, and is written $d_{1 m}$ and $d_{1 f}$ for males and females, respectively. In the next two sections, we derive estimates of dispersal at both levels based on a coancestry approach. The main symbols used throughout the model are summarized in the Appendix.

\section{Philopatry at the Population Level}

The coancestry between two individuals is defined as the probability that two alleles, randomly sampled at a given locus from these individuals, are identical by descent. Let $\gamma$ be the coancestry among two juveniles randomly sampled from the same breeding group, $\varphi$ the coancestry among two juveniles sampled in different breeding groups from the same population, and $\alpha$ the coancestry among juveniles from different populations.

The coancestry $\gamma_{m}$ among two adult males, sampled after dispersal in the same breeding group, is given by:

$$
\gamma_{m}=\gamma\left(1-x_{m}-z_{m}\right)+\varphi z_{m}+\alpha x_{m}
$$

where $x_{m}$ measures the probability that the two males stem from different populations, and $z_{m}$ the probability that they stem from different breeding groups of the same population. The coancestry among adult males sampled in different groups within the same population becomes:

$$
\varphi_{m}=\frac{z_{m}}{g-1} \gamma+\left(1-x_{m}-\frac{z_{m}}{g-1}\right) \varphi+x_{m} \alpha
$$

where $g$ is the number of breeding groups per population. Equation (1a) can be rewritten:

$$
z_{m}=\frac{\gamma\left(1-x_{m}\right)+\alpha x_{m}-\gamma_{m}}{\gamma-\varphi}
$$

and (1b):

$$
1-x_{m}=\frac{\varphi_{m}-\alpha}{\varphi-\alpha}-\frac{z_{m}}{g-1}\left(\frac{\gamma-\varphi}{\varphi-\alpha}\right) .
$$

Substituting (2a) in (2b), the probability that two males sampled in one population have the same population of origin becomes

$$
1-x_{m}=\frac{(g-1)\left(\varphi_{m}-\alpha\right)+\gamma_{m}-\alpha}{(g-1)(\varphi-\alpha)+\gamma-\alpha}
$$

or, equivalently, 


$$
1-x_{m}=\frac{\theta_{m}-\alpha}{\theta-\alpha}
$$

where $\theta=\gamma / g+\varphi[(g-1) / g]$ measures the coancestry among two juveniles randomly sampled from the same population, and $\theta_{m}=\gamma_{m} / g+\varphi_{m}[(g-1) / g]$ the corresponding parameter for adult males.

Assuming a large number of populations (and thus neglecting the possibility that two immigrant males have the same origin), then $1-x_{m}$ relates to dispersal as:

$$
1-x_{m}=\left(1-d_{2 m}\right)^{2} \text {. }
$$

Equating (3b) and (3c), and applying the same argument for females, we can estimate male and female dispersal among populations as:

$$
\hat{d}_{2 m}=1-\sqrt{\frac{\theta_{m}-\alpha}{\theta-\alpha}} \text { and } \quad \hat{d}_{2 f}=1-\sqrt{\frac{\theta_{f}-\alpha}{\theta-\alpha}} .
$$

As this result shows, breeding groups can be neglected when evaluating among-population dispersal, because the genetic consequences of $d_{2}$ do not depend on structures below the population level. The only parameters required are coancestries within and among populations.

Coancestries can also be expressed as functions of variance components, because, under our assumption of a large number of populations, $1-\alpha$ measures the total variance in the system $\left(\sigma_{t}^{2}\right)$ and $1-\theta$ the variance within local populations (Cockerham 1973). We thus obtain, for juveniles, $1-\alpha=$ $\sigma_{t}^{2}=\sigma_{w}^{2}+\sigma_{b}^{2}+\sigma_{a}^{2}$ and $1-\theta=\sigma_{w}^{2}+\sigma_{b}^{2}$, where $\sigma_{w}^{2}$ represents the variance component within individuals, $\sigma_{b}^{2}$ that between individuals within populations, and $\sigma_{a}^{2}$ that among populations. In adults, the total variance becomes $1-\alpha=\sigma_{t}^{2}=$ $\sigma_{w}^{2}+\sigma_{b_{m}}^{2}+\sigma_{a_{m}}^{2}=\sigma_{w}^{2}+\sigma_{b_{f}}^{2}+\sigma_{a_{f}}^{2}$, and that within populations $1-\theta_{m}=\sigma_{w}^{2}+\sigma_{b_{m}}^{2}$ and $1-\theta_{f}=\sigma_{w}^{2}+\sigma_{b_{f}}^{2}$ respectively, where $\sigma_{b_{m}}^{2}$ and $\sigma_{b_{f}}^{2}$ represent the within-population variance, and $\sigma_{a_{m}}^{2}$ and $\sigma_{a_{f}}^{2}$ the among-population variance in adult males and adult females, respectively. Note that neither the total variance $\left(\sigma_{t}^{2}\right)$, nor the variance within individuals $\left(\sigma_{w}^{2}\right)$, are affected by dispersal, because this process only transfers a proportion $x_{m}$ of the variance from the among-populations $\left(\sigma_{a}^{2}\right)$ to the between-individuals, within-populations $\left(\sigma_{b}^{2}\right)$ compartment. Thus, $\sigma_{b}^{2}+\sigma_{a}^{2}=\sigma_{b_{m}}^{2}+\sigma_{a_{m}}^{2}=\sigma_{b_{f}}^{2}+\sigma_{a_{f}}^{2}$ so that (4a) can be rewritten:

$$
\hat{d}_{2 m}=1-\sqrt{\frac{\sigma_{a_{m}}^{2}}{\sigma_{a}^{2}}} \text { and } \hat{d}_{2 f}=1-\sqrt{\frac{\sigma_{a_{f}}^{2}}{\sigma_{a}^{2}}} .
$$

Note that equation (4b) may also be written (Vitalis 2002):

$$
\hat{d}_{2 m}=1-\sqrt{\frac{F_{\mathrm{ST}_{m}}}{F_{\mathrm{ST}}}} \text { and } \hat{d}_{2 f}=1-\sqrt{\frac{F_{\mathrm{ST}_{f}}}{F_{\mathrm{ST}}}}
$$

where $F_{\mathrm{ST}_{f}}=\left(\theta_{m}-\alpha\right) /(1-\alpha)=\sigma_{a_{m}}^{2} / \sigma_{t}^{2}$ measures the genetic differentiation of adult males among populations, $F_{\mathrm{ST}_{m}}=\left(\theta_{f}\right.$ $-\alpha) /(1-\alpha)=\sigma_{a_{f}}^{2} / \sigma_{t}^{2}$ the corresponding statistics for adult females, and $F_{\mathrm{ST}}=(\theta-\alpha) /(1-\alpha)=\sigma_{a}^{2} / \sigma_{t}^{2}$ that for juveniles (Weir and Cockerham 1984).

\section{Philopatry at the Breeding Group Level}

Equation (1a) can be rewritten:

$$
1-x_{m}-z_{m}=\frac{\gamma_{m}-\varphi\left(1-x_{m}\right)-\alpha x_{m}}{\gamma-\varphi}
$$

and, using (2b):

$$
1-x_{m}-\frac{g}{g-1} z_{m}=\frac{\gamma_{m}-\varphi_{m}}{\gamma-\varphi} .
$$

If we assume $g$ is large (and thus neglect the possibility that two males immigrant from the neighborhood were born in the same group) then (5b) becomes:

$$
1-x_{m}-z_{m}=\frac{\gamma_{m}-\varphi_{m}}{\gamma-\varphi},
$$

Under the same assumption, the probability that two males sampled in one group originated from the same group is equivalent to philopatry at the group level:

$$
1-x_{m}-z_{m}=\left(1-d_{1 m}-d_{2 m}\right)^{2} \text {. }
$$

Combining (5c) and (5d) provides an estimate of total male dispersal:

$$
\hat{d}_{1 m}+\hat{d}_{2 m}=1-\sqrt{\frac{\gamma_{m}-\varphi_{m}}{\gamma-\varphi}} .
$$

The same reasoning for females provides:

$$
\hat{d}_{1 f}+\hat{d}_{2 f}=1-\sqrt{\frac{\gamma_{f}-\varphi_{f}}{\gamma-\varphi}} .
$$

Coancestries, again, can be expressed as functions of the variance components. The total variance is decomposed as $\sigma_{t}^{2}=\sigma_{w}^{2}+\sigma_{c}^{2}+\sigma_{g}^{2}+\sigma_{s}^{2}$, where $\sigma_{c}^{2}, \sigma_{g}^{2}$, and $\sigma_{s}^{2}$ measure respectively the within-group, the among-groups within populations, and the among-populations components. Note that these components relate to those obtained in the nonhierarchical analysis of the previous section as $\sigma_{a}^{2}=\sigma_{s}^{2}+(1 / g)$ $\sigma_{g}^{2}$ and $\sigma_{b}^{2}=\sigma_{c}^{2}+[(g-1) / g] \sigma_{g}^{2}$.

In this hierarchical framework, the within-populations variance for juveniles becomes $1-\varphi=\sigma_{w}^{2}+\sigma_{c}^{2}+\sigma_{g}^{2}$, and the within-groups component amounts to $1-\gamma=\sigma_{w}^{2}+\sigma_{c}^{2}$. The same decomposition can be applied to adult males and females, by noting that dispersal transfers a proportion $x_{m}$ (respectively $x_{f}$ ) of the among-population component $\left(\sigma_{s}^{2}\right)$, and a proportion $x_{m}+z_{m}$ (respectively, $x_{f}+z_{f}$ ) of the amonggroup component $\left(\sigma_{g}^{2}\right)$, to the within group component $\left(\sigma_{c}^{2}\right)$. As a result, (6a) and (6b) can be rewritten as:

$\hat{d}_{1 m}+\hat{d}_{2 m}=1-\sqrt{\frac{\sigma_{g_{m}}^{2}}{\sigma_{g}^{2}}}$ and $\quad \hat{d}_{1 f}+\hat{d}_{2 f}=1-\sqrt{\frac{\sigma_{g_{f}}^{2}}{\sigma_{g}^{2}}}$,

where $\sigma_{g m}^{2}$ and $\sigma_{g f}^{2}$ represent the variance among groups within populations, in adult males and females, respectively.

These quantities can similarly be expressed as functions of $F$-values ratios, as:

$$
\begin{aligned}
& \hat{d}_{1 m}+\hat{d}_{2 m}=1-\sqrt{\frac{F_{\mathrm{GT}_{m}}^{*}-F_{\mathrm{ST}_{m}}^{*}}{F_{\mathrm{GT}}^{*}-F_{\mathrm{ST}}^{*}}} \text { and } \\
& \hat{d}_{1 f}+\hat{d}_{2 f}=1-\sqrt{\frac{F_{\mathrm{GT}_{f}}^{*}-F_{\mathrm{ST}_{f}}^{*}}{F_{\mathrm{GT}}^{*}-F_{\mathrm{ST}}^{*}}}
\end{aligned}
$$


where astericks are meant to signal that these $F$-statistics are estimated hierarchically; that is,

$$
\begin{aligned}
& F_{\mathrm{ST}}^{*}=\frac{\varphi-\alpha}{1-\alpha}=\frac{\sigma_{s}^{2}}{\sigma_{t}^{2}}, \quad F_{\mathrm{ST}_{m}}^{*}=\frac{\varphi_{m}-\alpha}{1-\alpha}=\frac{\sigma_{s_{m}}^{2}}{\sigma_{t}^{2}}, \quad \text { and } \\
& F_{\mathrm{ST}_{f}}^{*}=\frac{\varphi_{f}-\alpha}{1-\alpha}=\frac{\sigma_{s_{f}}^{2}}{\sigma_{t}^{2}}
\end{aligned}
$$

measure the proportion of variance due to differentiation among populations in juveniles, adult males and adult females, respectively. Similarly,

$$
\begin{aligned}
& F_{\mathrm{GT}_{m}}^{*}=\frac{\gamma_{m}-\alpha}{1-\alpha}=\frac{\sigma_{g_{m}}^{2}+\sigma_{s_{m}}^{2}}{\sigma_{t}^{2}}, \\
& F_{\mathrm{GT}_{f}}^{*}=\frac{\gamma_{f}-\alpha}{1-\alpha}=\frac{\sigma_{g_{f}}^{2}+\sigma_{s_{f}}^{2}}{\sigma_{t}^{2}}, \text { and } \\
& F_{\mathrm{GT}}^{*}=\frac{\gamma-\alpha}{1-\alpha}=\frac{\sigma_{g}^{2}+\sigma_{s}^{2}}{\sigma_{t}^{2}}
\end{aligned}
$$

measure the proportion of total variance due to the differentiation among breeding groups in adult males, adult females, and juveniles, respectively. Equations (6a) to (6c) are indeed recovered by inserting these expressions for $F_{G \mathrm{~T}}^{*}$ and $F_{\text {ST }}^{*}$ into $(6 \mathrm{~d})$. Note also that $\hat{d}_{1 m}$ and $\hat{d}_{1 f}$ are readily obtained by subtracting equation (4) from (6).

\section{Hierarchical versus Nonhierarchical Analyses}

Although a hierarchical approach is obviously required to evaluate $d_{1}+d_{2}$ (previous section), a nonhierarchical approach is to be used when evaluating $d_{2}$. This is made evident by comparing equations ( $2 \mathrm{~b})$ and $(3 \mathrm{~b})$, which are fully equivalent equations. Although the ratio of nonhierarchical statistics $F_{\mathrm{ST}_{m}} / F_{\mathrm{ST}}=\sigma_{a_{m}}^{2} / \sigma_{a}^{2}=\left(\theta_{m}-\alpha\right) /(\theta-\alpha)$ entirely describes the right-hand sides of (3b), the ratio on hierarchical statistics $F_{\mathrm{ST}_{m}}^{*} / F_{\mathrm{ST}}^{*}=\sigma_{s_{m}}^{2} / \sigma_{s}^{2}=\left(\varphi_{m}-\alpha\right) /(\varphi-\alpha)$ only encompasses the first term in the right-hand side of (2b). Using this ratio would overestimate $1-x_{m}$ (and thus underestimate $d_{2}$ ). As our simulations revealed (see below), the bias introduced in that way can be significant if the number of groups is low, because $\sigma_{g}^{2}=\gamma-\varphi$ can largely exceed $\sigma_{s}^{2}=\varphi-\alpha$ in structured populations.

\section{INDiVIDUAL-BASEd Simulations}

In order to validate our approach, before applying it to our shrew dataset, we simulated several sets of genetic data using a version of Easypop (Balloux 2001) slightly modified to generate two output files. One file describes the genetic structure after dispersal for generation $t$ (parents), and the other the genetic structure before dispersal for generation $t+1$ (offspring). Parameter values were chosen to fit our biological model and sampling design. Thus, we implemented seven unlinked loci with a mutation rate $\mu=10^{-4}$ (KAM, with $\mathrm{K}$ $=30$ ), a monogamous mating system, and a hierarchical island structure. Forty breeding groups were nested into local populations according to three different hierarchical designs: (A) 20 populations $\times 2$ groups; (B) eight populations $\times$ five groups; and $(\mathrm{C})$ five populations $\times$ eight groups. Dispersal rates between populations $\left(d_{2}\right)$ and between groups within populations $\left(d_{1}\right)$ were varied from 0 to 0.2 (step 0.1$)$ for each sex independently, in 20 different combinations (of 81 feasible) chosen so as to balance the design (number of simulations for the different values of $d_{1}, d_{2}$ and $d_{1}+d_{2}$ ), and to include the most contrasted situations. Situations symmetrical with respect to sex were dropped, because we were considering a monogamous mating system. Regarding population and sample sizes, a first scenario (denoted 5/5), applied to all three hierarchical designs (A, B, and C), consisted of five individuals sampled of five present (small groups, exhaustive sampling). Two additional scenarios were applied to the $8 \times 5$ design, namely a scenario $5 / 25$, with five individuals sampled of 25 present (large groups, partial sampling), and a scenario 25/25, with 25 individuals sampled of 25 present (large groups, exhaustive sampling). In all cases, sample sizes before dispersal (offspring) and after dispersal (parents) were the same. We performed 400 simulations for each of the five patterns of hierarchical design and sampling scenarios (20 different combinations of dispersal rates $\times 20$ replicates each), except for B25 of 25 , for which simulations were highly time consuming, so that only 200 runs were performed (10 combinations $\times 20$ replicates).

For each of these 1800 simulations, variance components were extracted at equilibrium for adult males and females (postdispersal) as well as juveniles (predispersal), to estimate $\hat{d}_{2}$ (eq. 4b) and $\hat{d}_{1}+\hat{d}_{2}$ (eq. 6c), using the MINQUE (Minimum Norm Quadratic Unbiased Estimation) procedure (Rao 1971, 1972) implemented in S 2000 (Mathsoft, Inc., Seattle, WA). Estimates over loci and alleles were combined following Weir and Cockerham (1984). Dispersal parameters were also computed from $F$-statistics. These two alternative approaches ( $F$-statistics vs. variance components) provided very similar values, because the total variance had very similar values in the juvenile and the adult samples (as assumed in our derivation). As justified in the previous section, the estimator $\hat{d}_{1}+\hat{d}_{2}$ was evaluated through a full (three-levels) hierarchical analysis, whereas the group level of genetic structure was dropped when evaluating $\hat{d}_{2}$. Indeed, our simulation results confirmed that $d_{2}$ was systematically and significantly underestimated when using the among-population component of variance obtained from a full (three-level) hierarchical approach (results not shown). This bias disappeared when using results from the two-level analysis.

For given $d_{i}$ values, the estimates $\hat{d}_{i}$ did not differ among males and females (Wilcoxon signed-rank test, $Z=-1.134$, $P=0.27$ ), so results will be given without regard to sex. Estimators appear reasonably good (Fig. 1), but tend to slightly overestimate the true parameter values under certain circumstances, with the median of discrepancies $\left(\hat{d}_{i}-d_{i}\right)$ averaging +0.02 . Population and sample sizes affect the performances of the three estimators (Fig. 1). Standard deviations of discrepancies are lowest in scenario 25/25 (SD = $0.08)$, and highest in scenario $5 / 25(\mathrm{SD}=0.29)$.

Results for scenario $5 / 5$ across the three hierarchical designs (A, B, and C) are provided in Figure 2. Standard deviations of discrepancies increase with dispersal rate (Fig. 2b-d). The estimator $\hat{d}_{2}$ performs worst (high variance and slight overestimate) when the number of populations is low $(5 \times 8$; Fig. $2 \mathrm{~b})$, while $\hat{d}_{1}+\hat{d}_{2}$ performs worst when the 


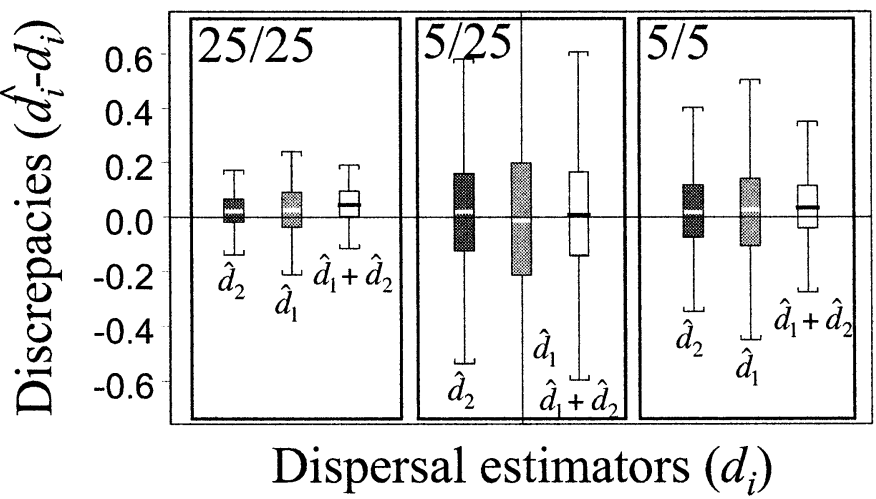

FIG. 1. Discrepancies $\left(\hat{d}_{i}-d_{i}\right)$ between estimated and true dispersal rates for the three estimators $\left(\hat{d}_{2}, \hat{d}_{1}+\hat{d}_{2}\right.$ and $\left.\hat{d}_{1}\right)$ across the three sampling scenarios: 25/25 (large groups, exhaustive sampling); 5/25 (large groups, partial sampling); and 5/5 (small groups, exhaustive sampling). Simulations were performed for the hierarchical design $8 \times 5$ ( 8 populations $\times 5$ groups $)$. Boxplots with median, first quartile and standard span $(1.5 \times$ interquartile range $)$.

number of groups per population is low $(20 \times 2$; Fig. $2 d)$. As a result, the intermediate pattern $(8 \times 5)$ offers the best situation in our simulations.

Table 1 provides an analysis of variance (ANOVA) detailing the effects of the two dispersal rates $d_{1}$ and $d_{2}$ (and their interaction) on the discrepancies in estimators ( $P$-values based on 2000 randomizations; Manly 1997). Significant effects are mostly restricted to the $20 \times 2$ pattern. Although the performance of $\hat{d}_{2}$ depends only on the value of $d_{2}$ (decreasing as $d_{2}$ increases), the performance of $\hat{d}_{1}+\hat{d}_{2}$ (and hence that of $\hat{d}_{1}$ ) depends on $d_{1}, d_{2}$, and on their interaction.

\section{EMPIRICAL DATASET}

The greater white-toothed shrew, C. russula, is a small (11$14 \mathrm{~g}$ ) insectivorous mammal, widespread in southern and central Europe. The species is anthropophilic in the northern part of its distribution (including the study area) where it occurs in discrete populations inhabiting villages and suburbs. Populations are further structured into smaller groups of a few breeding pairs (Balloux et al. 1998), linked to thermally favorable sites (farms, stables, compost piles in gardens) required to meet the energetic needs of the cold season (Genoud and Hausser 1979).

The study area is situated in western Switzerland, between the Lake Leman and the Jura mountains region ("La Côte" region). A hierarchical sampling was performed from June to August in 1999 and 2000. A total of 49 gardens (breeding groups) within 15 villages (populations) were sampled in an area of $12 \times 12 \mathrm{~km}$ (see Ehinger et al. 2002 for precise localization and trapping details). Neighboring villages are separated by $3.3 \mathrm{~km}$ on average, and gardens ( 3.3 per village on average) by about $0.4 \mathrm{~km}$. In each garden, an average of 3.5 predispersal juveniles (range $0-9$ ), 3.7 adult females (range 1-9), and 3.8 adult males (0-12) were trapped. This amounts to 536 individuals, including 185 adult males, 181 adult females, and 170 juveniles, recognized by their grayish coloration, lack of teats (females) or lateral glands (males), and lighter weight ( $\leq 9 \mathrm{~g})$. Tissue samples were collected by toe clipping, then stored at $-20^{\circ} \mathrm{C}$. Individuals were released onsite after trapping.

Total DNA was extracted from frozen phalanges following a salt/chloroform procedure modified from Miller et al. (1988) by adding one step of chloroform/isoamylalcohol extraction (24/1). All individuals were scored for seven microsatellite loci, including the loci 3, 9, 54, 57, and 72 of Favre and Balloux (1997) plus the loci 52 and 82 (Genebank nos. AY034429 and AY034430). Amplifications were run on a $6 \%$ polyacrylamide gel on an ABI 377 sequencer (Applied Biosystems Foster City, CA). Hierarchical and nonhierarchical $F$-statistics were computed as above from the variance components, with $95 \%$ confidence intervals obtained by bootstrapping over loci. These values were then used to compute estimators of dispersal rates with $95 \%$ confidence intervals (Table 2).

Results show that $F_{\mathrm{ST}^{-}}$values (differentiation among villages) do not differ significantly among sexes (randomization test, Goudet 1995; Goudet et al. 2002), whereas $F_{\mathrm{GT}}$-values (differentiation among gardens) are significantly stronger in males (randomization test, $P<0.05$, Goudet 1995; Goudet et al. 2002). As a result, $\hat{d}_{2}$ estimators do not differ significantly among sexes, while $\hat{d}_{1}+\hat{d}_{2}$ are significantly female biased. $F$ Gैs-values (differentiation among gardens within villages) are significantly higher in males (Wilcoxon's signedrank, $P<0.01)$ and consequently, $\hat{d}_{1}$ are also significantly female biased. An estimate of $38 \%$ of breeding females are immigrant, $14 \%$ stemming from other groups within the same village (local dispersal) and 24\% from other villages (longdistance dispersal). All these dispersal-rate values differ significantly from zero. In males, by contrast, dispersal among villages $(15 \%)$ is the only significant parameter, dispersal among groups being close to (and not significantly different from) zero.

Our genetic data displayed a strong isolation by distance. Regressions of pairwise $F_{\mathrm{ST}} /\left(1-F_{\mathrm{ST}}\right)$ on the logarithm of geographical distances (in meters) among villages (Rousset 1997) revealed significant regression coefficients for the three classes of individuals (adult males: $\beta=0.022, r^{2}=0.17, P$ $<0.001$; adult females: $\beta=0.024, r^{2}=0.20, P<0.001$; juveniles: $\beta=0.029, r^{2}=0.18, P<0.001$; Mantel tests). As our derivation of parameter estimators assumed an island model of dispersal, the question arises, whether isolation by distance might affect our estimates of dispersal rates. However, though pairwise $F_{\text {ST }}$ are indeed lower among neighboring villages than among random villages, the net effect on dispersal estimate is likely to be weak, because both adult and juvenile $F_{\mathrm{ST}}$ are similarly affected. In order to check this point, we also estimated dispersal rates from pairwise $F$ statistics (Rousset 2000), selecting pairs of villages connected by Delaunay tesselation (a connection network based on proximity criteria; Brassel and Reif 1979). The resulting dispersal estimates indeed show very little change from those based on the whole-population $F$-statistics, amounting to $\hat{d}_{2 m}$ $=0.12$ and $\hat{d}_{2 f}=0.24$ among villages, $\hat{d}_{1 m}=-0.01$ and $\hat{d}_{1 f}$ $=0.14$ among gardens within villages, and $\hat{d}_{1 m}+\hat{d}_{2 m}=0.11$ and $\hat{d}_{1 f}+\hat{d}_{2 f}=0.38$ for total dispersal.

\section{DISCUSSION}

$F$-statistics have long been used to estimate dispersal (Wright 1943). In this classical use, effective dispersal rates 
a)

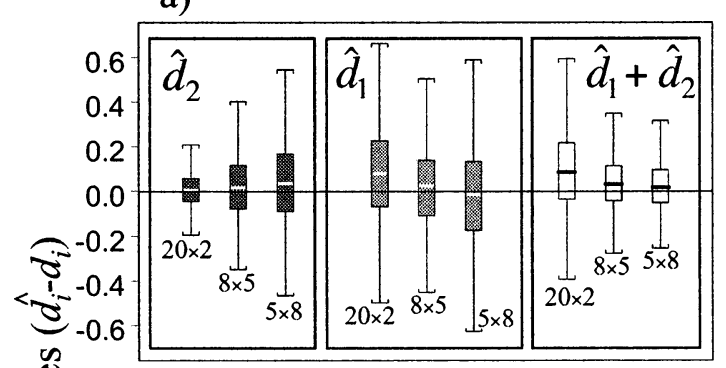

Hierarchical design

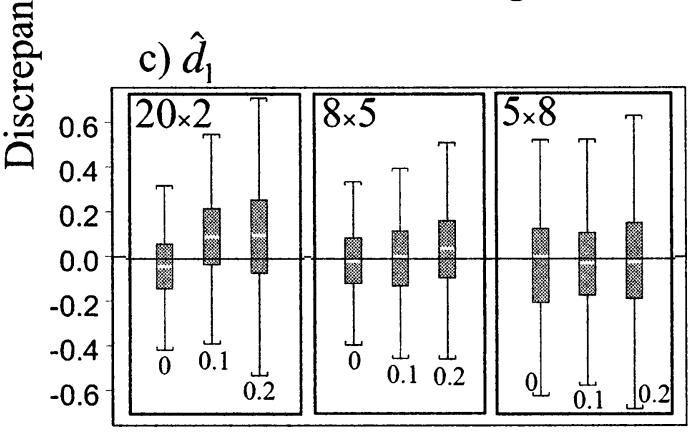

True dispersal rate $\left(d_{1}\right)$ b) $d_{2}$

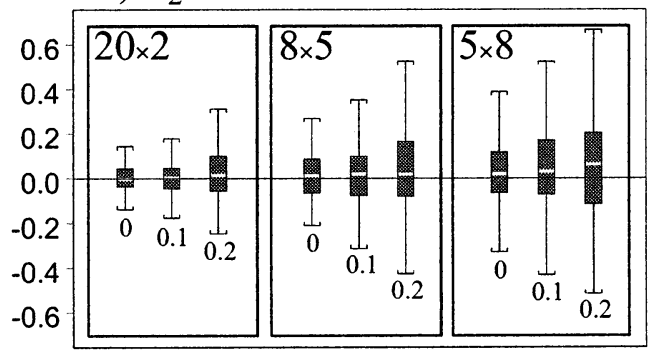

True dispersal rate $\left(d_{2}\right)$

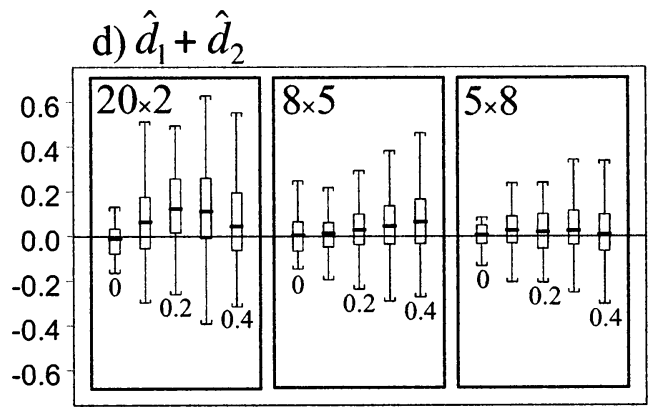

True dispersal rate $\left(d_{1}+d_{2}\right)$

FIG. 2. Discrepancies $\left(\hat{d}_{i}-d_{i}\right)$ between estimated and true dispersal rates for the three estimators $\left(\hat{d}_{2}, \hat{d}_{1}+\hat{d}_{2}\right.$ and $\left.\hat{d}_{1}\right)$ across the three hierarchical designs $20 \times 2(20$ populations $\times 2$ breeding groups $), 8 \times 2$, and $5 \times 8$. The simulations were performed for the sampling scenario $5 / 5$ (small groups, exhaustive sampling). Boxplots with median, first quartile and standard span $(1.5 \times$ interquartile range).

are estimated through the genetic differentiation of populations, a measure of the history of gene flow among them. This approach has been criticized for being too sensitive to the underlying assumptions, among which are equilibrium dynamics and island structure (Wade and McCauley 1988; Whitlock and McCauley 1999). The present approach, pioneered by Vitalis (2002), differs from the classical one by relying, not on the absolute $F_{\mathrm{ST}}$-value, but on the contrast among sexes and age classes. Accordingly, estimates appear less sensitive to departures from the above assumptions. Isolation by distance had only slight effect on our dispersal estimates, presumably because both adult and juvenile $F_{\text {ST }}$ are simultaneously affected by distance. Disequilibrium dynamics should not matter significantly either, because our estimates reflect instantaneous, rather than historical, rates of dispersal. In other words, our approach detects the presence of actual immigrants in the sample, not the contribution of past immigrants to the local gene pool. The only effect of past dispersal is to homogenize gene pools, and thereby to decrease the power of the present analysis. It is worth noting that instantaneous dispersal might differ from effective dispersal if the genetic contribution of immigrants to local gene pool differs from average.

From our individual-based simulations, the approach proposed here allows fairly good estimates of dispersal in hierarchically structured populations. However, true dispersal rates appear slightly overestimated under certain circumstances. This bias, already noticed by Vitalis (2002), most likely stems from our infinite-island assumptions. Our formalization neglects the possibility that two migrants may come from the same natal group or population. This induces a slight bias in $\hat{d}_{2}$ when the number of subpopulations is

TABLE 1. Effects of hierarchical design and true dispersal rates on the performances of estimators $\left(\hat{d}_{2}, \hat{d}_{1}+\hat{d}_{2}\right.$ and $\left.\hat{d}_{1}\right)$. ANOVAs with $P$-values based on 2000 randomizations. Significant $P$-values are in bold.

\begin{tabular}{|c|c|c|c|c|c|c|c|c|}
\hline \multirow{2}{*}{$\begin{array}{l}\text { Hier- } \\
\text { archical } \\
\text { design }\end{array}$} & \multirow{2}{*}{$\begin{array}{c}\text { Source of } \\
\text { variation }\end{array}$} & \multirow[b]{2}{*}{$\mathrm{df}$} & \multicolumn{2}{|c|}{$\hat{d}_{2}$} & \multicolumn{2}{|c|}{$\hat{d}_{1}+\hat{d}_{2}$} & \multicolumn{2}{|c|}{$\hat{d}_{1}$} \\
\hline & & & MS & $P$-value & MS & $P$-value & MS & $P$-value \\
\hline \multirow{4}{*}{$20 \times 2$} & $d_{1}$ & 2 & 0.009 & 0.469 & 0.611 & 0 & 0.685 & 0 \\
\hline & $d_{2}$ & 2 & 0.062 & 0.003 & 0.277 & 0 & 0.622 & 0 \\
\hline & $d_{1}: d_{2}$ & 4 & 0.002 & 0.977 & 0.119 & 0.019 & 0.130 & 0.027 \\
\hline & $d_{1}$ & 2 & 0.019 & 0.508 & 0.290 & 0 & 0.165 & 0.021 \\
\hline \multirow[t]{2}{*}{$8 \times 5$} & $d_{2}$ & 2 & 0.047 & 0.144 & 0.009 & 0.622 & 0.017 & 0.666 \\
\hline & $d_{1}: d_{2}$ & 4 & 0.052 & 0.359 & 0.005 & 0.171 & 0.014 & 0.865 \\
\hline \multirow{3}{*}{$5 \times 8$} & $d_{1}$ & 2 & 0.001 & 0.989 & 0.039 & 0.088 & 0.034 & 0.704 \\
\hline & $d_{2}$ & 2 & 0.041 & 0.551 & 0.002 & 0.881 & 0.047 & 0.585 \\
\hline & $d_{1}: d_{2}$ & 4 & 0.052 & 0.535 & 0.005 & 0.870 & 0.066 & 0.546 \\
\hline
\end{tabular}


TABLE 2. F-statistics and sex-specific dispersal rates (in bold) for the Crocidura russula dataset. Confidence intervals (95\%) in brackets. Asterisks denote hierarchical analyses.

\begin{tabular}{|c|c|c|c|c|c|c|c|}
\hline & \multirow[b]{2}{*}{$n$} & \multicolumn{2}{|c|}{$\begin{array}{c}\text { Non-hierarchical } \\
\text { analysis }\end{array}$} & \multicolumn{3}{|c|}{$\begin{array}{l}\text { Hierarchical } \\
\text { analysis }\end{array}$} & \multirow[b]{2}{*}{$\hat{d}_{1}$} \\
\hline & & $F_{\mathrm{ST}}$ & $\hat{d}_{2}$ & $F_{S T}^{*}$ & $F_{\mathrm{GT}}^{*}$ & $\hat{d}_{1}+\hat{d}_{2}$ & \\
\hline Juveniles & 170 & $\begin{array}{c}0.090 \\
{[0.077 ; 0.104]}\end{array}$ & & $\begin{array}{c}0.047 \\
{[0.031 ; 0.063]}\end{array}$ & $\begin{array}{c}0.153 \\
{[0.140 ; 0.167]}\end{array}$ & & \\
\hline Adult females & 181 & $\begin{array}{c}0.053 \\
{[0.047 ; 0.059]}\end{array}$ & $\begin{array}{c}\mathbf{0 . 2 4} \\
{[0.18 ; 0.29]}\end{array}$ & $\begin{array}{c}0.040 \\
{[0.034 ; 0.046]}\end{array}$ & $\begin{array}{c}0.081 \\
{[0.072 ; 0.088]}\end{array}$ & $\begin{array}{c}\mathbf{0 . 3 8} \\
{[0.30 ; 0.48]}\end{array}$ & $\begin{array}{c}\mathbf{0 . 1 4} \\
{[0.08 ; 0.21]}\end{array}$ \\
\hline Adult males & 185 & $\begin{array}{c}0.065 \\
{[0.052 ; 0.080]}\end{array}$ & $\begin{array}{c}\mathbf{0 . 1 5} \\
{[0.05 ; 0.25]}\end{array}$ & $\begin{array}{c}0.035 \\
{[0.025 ; 0.047]}\end{array}$ & $\begin{array}{c}0.121 \\
{[0.097 ; 0.143]}\end{array}$ & $\begin{array}{c}\mathbf{0 . 1 0} \\
{[-0.02 ; 0.25]}\end{array}$ & $\begin{array}{c}-\mathbf{0 . 0 5} \\
{[-0.15 ; 0.06]}\end{array}$ \\
\hline
\end{tabular}

small (as low as five in Fig. 2b, 3rd panel) and in $\hat{d}_{1}$ when the number of groups per subpopulation is small (as low as two in Fig. 2c-d, 1st panel). However, although the assumption was grossly violated, the bias appears really weak.

The variance of estimates was in some cases quite large, which can be attributed to several reasons. First, the variance is higher for $\hat{d}_{1}$ (Fig. 1) because this estimator depends on the two others, and thus combines their measurement errors. Second, the variance is higher when samples are small (Fig. 1 ). As shown by Goudet (1993), the variance of $F$-statistics estimates tends to increase as sample size decreases. The effect is the more pronounced here that we are dealing with ratios of $F$-statistics. Third, exhaustive samples do better than partial samples (compare $5 / 5$ and 5/25 in Fig. 1). As already noted, our method works only insofar as immigrants are effectively included in the sample. Nonexhaustive sampling opens the possibility that immigrants are over- or underrepresented relative to their actual occurrence, and thereby introduces an additional sampling variance in the estimate. Finally, the apportionment of sampling effort among the several hierarchical levels plays a role. The variance is smallest for $\hat{d}_{2}$ when the number of populations is highest $(20 \times 2)$, and for $\hat{d}_{1}+\hat{d}_{2}$ when the number of groups per population is highest $(5 \times 8)$.

The performance of $\hat{d}_{2}$ appears independent of the value of $d_{1}$ (Table 1 ), as long as a nonhierarchical analysis is performed, so that substructures can be safely ignored when estimating dispersal at a large geographical scale. By contrast, $\hat{d}_{1}$ does depend on $d_{2}$, so that large-scale structures cannot be ignored. In other words, the variance among groups within populations also depends on the level of immigration from other populations, so that the dispersal rate among groups within populations might be largely overestimated if the analysis fails to recognize long-distance immigrants.

From the above results, some suggestions can be drawn concerning the optimal sampling strategy. The sampling design should include the highest hierarchical level at which dispersal is suspected to occur (here, populations), the number of groups per population should approximate the number of populations in the sample, and sampling should be as exhaustive as possible within groups. Note that a similar conclusion was reached by Goudet et al. (2002) concerning the power of statistical tests designed to detect sex biases in dispersal.

Our empirical dataset confirms that dispersal is female biased in C. russula, as previously evidenced by Favre et al. (1997). The total dispersal rate of females is close to $40 \%$, that is, three to four times higher than that of males (10$15 \%)$. A very similar estimate (39\% female dispersal) was obtained by Balloux et al. (1998), using an alternative approach based on Chesser's (1991b) recurrence equations. However, these authors, assumed complete philopatry for males, because the approach they used did not allow simultaneous estimation of the two parameters. From our present approach, this latter assumption appears to hold true at the local scale, but not at the larger geographical scale (among villages), because $10-15 \%$ of males appear to be long-distance immigrants. In females, by contrast, dispersal is not significantly stronger at long distance than at short distance. Our quantitative estimates of dispersal seem robust regarding our assumptions on the mode of dispersal (island vs. stepping stone), because the $F$-statistics computed on pairs of neighboring villages provided basically the same figures.

These results suggest that, in $C$. russula, distinct selective forces drive dispersal at different distances. This pattern might actually be quite widespread. As Ronce et al. (2001, p.345) put it: “... distances travelled to colonize newly opened habitats are expected to be larger than those travelled to avoid interactions with relatives.' Indeed, long-distance movements are likely to respond to crowding conditions or (re-) colonization opportunities, so that selective pressures at this scale are not expected to differ among sexes. By contrast, local dispersal is likely to respond to local interactions. Short-distance movements should suffice to escape inbreeding or kin competition, two forces most likely to induce sex biases in dispersal (Perrin and Goudet 2001). In the present instance, inbreeding avoidance can be ruled out as a significant force behind dispersal (Bouteiller and Perrin 2000; Duarte et al. 2003). Local resource competition (in females) and local resource enhancement (in males) are the most likely alternatives.

This paper provides the first empirical validation of Vitalis (2002) approach to estimate dispersal, based on a contrast of sex- and age-specific components of genetic variance. Furthermore, it shows that this approach can be readily extended to account for the hierarchical structure of natural populations. We think this approach has a great potential of application, because the selective forces on dispersal in nature are likely to vary with the spatial scale considered, so that sexbiases in dispersal, a widespread feature of breeding systems, are also likely to depend on spatial scale in a wide range of ecological contexts. 


\section{ACKNOWLEDGMENTS}

We want to thank F. Balloux for the modified version of Easypop, J. Goudet for statistical advice; C. Bouteiller, J. Goudet, L. Lehmann, and M. Reuter for helpful discussions; and the Swiss National Science Foundation for financial support (grant no. 31-55475.98 to NP and EP).

\section{Literature Cited}

Balloux, F. 2001. EASYPOP (version 1.7): a computer program for population genetics simulations. J. Hered. 92:301-302.

Balloux, F., J. Goudet, and N. Perrin. 1998. Breeding system and genetic variance in the monogamous, semi-social shrew, Crocidura russula. Evolution 52:1230-1235.

Bennetts, R. E., J. D. Nichols, J.-D. Lebreton, R. Pradel, J. E. Hines, and W. K. Kitchens. 2001. Methods for estimating dispersal probabilities and related parameters using marked animals. Pp. 3-17 in J. Clobert, E. Danchin, A. A. Dhondt, and J. D. Nichols, eds. dispersal. Oxford Univ. Press, New York.

Blundell, G. M., M. Ben-David, P. Groves, R. T. Bowyer, and E. Geffen. 2002. Characteristics of sex-biased dispersal and gene flow in coastal river otters: implications for natural recolonization of extirpated populations. Mol. Ecol. 11:289-303.

Bouteiller, C., and N. Perrin. 2000. Individual reproductive success and effective population size in the greater white-toothed shrew Crocidura russula. Proc. R. Soc. Lond. B Biol. Sci. 267:701-705.

Brassel, K. E., and D. Reif. 1979. Procedure to generate Thiessen polygons. Geogra. Anal. 11:289-303.

Chesser, R. K. 1991a. Influence of gene flow and breeding tactics on gene diversity within populations. Genetics 129:573-583. _ 1991b. Gene diversity and female philopatry. Genetics 127: 437-447.

Chesser, R. K., O. E. Rhodes, D. W. Sugg, and A. Schnabel. 1993a. Effective sizes for subdivided populations. Genetics 135: 1221-1232.

Chesser, R. K., D. W. Sugg, O. E. Rhodes, J. M. Novak, and M. H. Smith. 1993b. Evolution of mammalian social-structure. Acta Theriol. 38:163-174.

Clarke, A. L., B. E. Saether, and E. Roskaft. 1997. Sex biases in avian dispersal: a reappraisal. Oikos 79:429-438.

Clobert, J., E. Danchin, A. A. Dhondt, and J. D. Nichols. 2001. Dispersal. Oxford Univ. Press, New York.

Cockerham, C. C. 1973. Analyses of gene frequencies. Genetics 74:679-700.

Dobson, F. S. 1982. Competition for mates and predominant juvenile male dispersal in mammals. Anim. Behav. 30:1183-1192.

Dobson, F. S. 1994. Measures of gene flow in the Columbian ground-squirrel. Oecologia 100:190-195.

- 1998. Social structure and gene dynamics in mammals. J. Mammal. 79:667-670.

Duarte, L. C., C. Bouteiller, P. Fontanillas, E. Petit, and N. Perrin. 2003. Inbreeding in the greater white-toothed shrew, Crocidura russula. Evolution 57:638-645.

Ehinger, M., P. Fontanillas, E. Petit, and N. Perrin. 2002. Mitochondrial DNA variation along an altitudinal gradient in the greater white-toothed shrew, Crocidura russula. Mole. Ecol. 11: 939-945.

Favre, L., and F. Balloux. 1997. Characterization of microsatellite loci in the greater white-toothed shrew Crocidura russula. Mole. Ecol. 6:595-596.

Favre, L., F. Balloux, J. Goudet, and N. Perrin. 1997. Female-biased dispersal in the monogamous mammal Crocidura russula: evidence from field data and microsatellite patterns. Proc. R. Soc. Lond. B Biol. Sci. 264:127-132.

Gandon, S. 1999. Kin competition, the cost of inbreeding and the evolution of dispersal. J. Theor. Biol. 200:345-364.

Gandon, S., and Y. Michalakis. 2001. Multiple cause of the evolution of dispersal. Pp. 169-179 in J. Clobert, E. Danchin, A. A. Dhondt and J. D. Nichols, eds. Dispersal. Oxford Univ. Press, New York.
Genoud, M., and J. Hausser. 1979. Ecology of a Crocidura russula population in a rural mountain habitat. Terre Vie 33:539-554.

Goudet, J. 1993. The genetics of geographically structured populations. PhD, University of Wales.

. 1995. FSTAT (Vers. 1.2): a computer program to calculate $F$-statistics. J. Hered. 86:485-486.

Goudet, J., N. Perrin, and P. Waser. 2002. Tests for sex-biased dispersal using bi-parentally inherited genetic markers. Mol. Ecol. 11:1103-1114.

Greenwood, P. J. 1980. Mating systems, philopatry and dispersal in birds and mammals. Anim. Behav. 28:1140-1162.

Hanski, I. 2001. Population dynamic consequences of dispersal in locals populations and in metapopulation. Pp. 283-298 in J. Clobert, E. Danchin, A. A. Dhondt, and J. D. Nichols, eds. Dispersal. Oxford Univ. Press, New York.

Ji, W., S. D. Sarre, N. Aitken, R. K. S. Hankin, and M. N. Clout. 2001. Sex-biased dispersal and density-independent mating system in the Australian brushtail possum, as revealed by minisatellite DNA profiling. Mol. Ecol. 10:1527-1537.

Koenig, W. D., D. VanVuren, and P. N. Hooge. 1996. Detectability, philopatry, and the distribution of dispersal distances in vertebrates. Trends Ecol. Evol. 11:514-517.

Lambin, X., J. Aars, and S. B. Piertney. 2001. Dispersal, intraspecific competition, kin competition and kin facilitation: a review of empirical evidence. Pp. 110-122 in J. Clobert, E. Danchin, A. A. Dhondt, and J. D. Nichols, eds. Dispersal. Oxford Univ. Press, New York.

Manly, B. F. J. 1997. Bootstrap, randomisation, and Monte Carlo methods in biology. Chapman and Hall, London.

Miller, S. A., D. D. Dykes, and H. F. Polesky. 1988. A simple salting out procedure for extracting DNA from human nucleated cells. Nucleic Acids Res. 16:1215-1215.

Mossman, C. A., and P. M. Waser. 1999. Genetic detection of sexbiased dispersal. Mol. Ecol. 8:1063-1067.

Motro, U. 1991. Avoiding inbreeding and sibling competition-the evolution of sexual dimorphism for dispersal. Am. Nat. 137: $108-115$

- 1994. Evolutionary and continuous stability in asymmetric games with continuous strategy sets- the parental investment conflict as an example. Am. Nat. 144:229-241.

Murrell, D. J., J. M. J. Travis, and C. Dytham. 2002. The evolution of dispersal distance in spatially-structured populations. Oikos 97:229-236.

O'Rian, M. J., and S. Braude. 2001. Inbreeding versus outbreeding in captive and wild populations of naked mole-rats. Pp. 143154 in J. Clobert, E. Danchin, A. A. Dhondt, and J. D. Nichols, eds. Dispersal. Oxford Univ. Press, New York.

Paetkau, D., W. Calvert, I. Stirling, and C. Strobeck. 1995. Microsatellite analysis of population-structure in Canadian polar bears. Mol. Ecol. 4:347-354.

Peacock, M. M., and C. Ray. 2001. Dispersal in pikas (Ochotona princeps): combining genetic and demographic approaches to reveal spatial and temporal patterns. Pp. 43-56 in J. Clobert, E. Danchin, A. A. Dhondt, and J. D. Nichols, eds. Dispersal. Oxford Univ. Press, New York.

Perrin, N., and J. Goudet. 2001. Inbreeding, kinship, and the evolution of natal dispersal. Pp. 123-142 in J. Clobert, E. Danchin, A. A. Dhondt, and J. D. Nichols, eds. Dispersal. Oxford Univ. Press, New York.

Perrin, N., and V. Mazalov. 1999. Dispersal and inbreeding avoidance. Am. Nat. 154:282-292.

- 2000. Local competition, inbreeding, and the evolution of sex-biased dispersal. Am. Nat. 155:116-127.

Petit, E., F. Balloux, and J. Goudet. 2001. Sex-biased dispersal in a migratory bat: a characterization using sex-specific demographic parameters. Evolution 55:635-640.

Petit, E., F. Balloux, and L. Excoffier. 2002. Mammalian population genetics: why not Y? Trends Ecol. Evol. 17:28-33.

Prugnolle, F., and T. de Meeus. 2002. Inferring sex-biased dispersal from population genetic tools: a review. Heredity 88:161-165.

Pusey, A., and M. Wolf. 1996. Inbreeding avoidance in animals. Trends Ecol. Evol. 11:201-206. 
Pusey, A. E. 1987. Sex-biased dispersal and inbreeding avoidance in birds and mammals. Trends Ecol. Evol. 2:295-299.

Rand, D. M. 1994. Thermal habit metabolic-rate and the evolution of mitochondrial-DNA. Trends Ecol. Evol. 9:238-238.

- 2001. The units of selection on mitochondrial DNA. Annual Review of Ecol. Syst. 32:415-448.

Rao, C. R. 1971. Minque estimation of variance and covariance components. Ann. Math. Stat. 42:1477.

1972. Estimation of variance and covariance components in linear models. J. Am. Statistical Association 67:112-115.

Ronce, O., I. Olivieri, J. Clobert, and E. Danchin. 2001. Perspectives on the study of dispersal evolution. Pp. 341-357 in J. Clobert, E. Danchin, A. A. Dhondt, and J. D. Nichols, eds. Dispersal. Oxford Univ. Press, New York.

Rousset, F. 1997. Genetic differentiation and estimation of gene flow from $F$ - statistics under isolation by distance. Genetics 145 : 1219-1228.

. 2000. Inferences from spatial population genetics. Pp. 239269 in D. J. Balding, M. Bishop and C. Cannigs, eds. Handbook of statistical genetics. John Wiley, Chichester, U.K.

. 2001. Genetic approaches to the estimation of dispersal rates. Pp. 18-28 in J. Clobert, E. Danchin, A. A. Dhondt and J. D. Nichols, eds. Dispersal. Oxford Univ. Press, New York.

Rousset, F., and S. Gandon. 2002. Evolution of the distribution of dispersal distance under distance-dependent cost of dispersal. J. Evol. Biol. 15:515-523.

Slatkin, M. 1985. Gene flow in natural populations. Annu. Rev. Ecol. Syst. 16:393-430.

Slatkin, M., and L. Voelm. 1991. $F_{\mathrm{ST}}$ in a hierarchical island model. Genetics 127:627-629.

Sugg, D. W., R. K. Chesser, F. S. Dobson, and J. L. Hoogland. 1996. Population genetics meets behavioral ecology. Trends Ecol. Evol. 11:338-342.

vanVuren, D., and K. B. Armitage. 1994. Reproductive success of colonial and noncolonial female yellow-bellied marmots (Marmota-Flaviventris). J. Mammal. 75:950-955.

Vigouroux, V., and D. Couvet. 2000. The hierarchical island model revisited. Genet. Sel. Evol. 32:395-402.

Vitalis, R. 2002. Sex-specific genetic differentiation and coalescence times: estimating sex-biased dispersal rates. Mol. Ecol. $11: 125-138$.

Wade, M. J., and D. E. McCauley. 1988. Extinction and recolonization: their effects on the genetic differentiation of localpopulations. Evolution 42:995-1005.

Wang, J. L. 1999. Effective size and F-statistics of subdivided populations for sex-linked loci. Theore. Popul. Biol. 55:176-188.

Weir, B. S., and C. C. Cockerham. 1984. Estimating F-statistics for the analysis of population-structure. Evolution 38:1358-1370.

Whitlock, M. C. 2001. Dispersal and the genetic properties of metapopulations. Pp. 273-282 in J. Clobert, E. Danchin, A. A. Dhondt, and J. D. Nichols, eds. Dispersal. Oxford Univ. Press, New York.

Whitlock, M. C., and D. E. McCauley. 1999. Indirect measures of gene flow and migration: $F_{\mathrm{ST}} \neq 1 /(4 \mathrm{Nm}+1)$. Heredity 82: $117-125$.

Wiens, J. A. 2001. Landscape context of dispersal. Pp. 96-109 in J. Clobert, E. Danchin, A. A. Dhondt, and J. D. Nichols, eds. Dispersal. Oxford Univ. Press, New York.

Wolff, J. O., K. I. Lundy, and R. Baccus. 1988. Dispersal, inbreeding avoidance and reproductive success in white-footed mice. Anim. Behav. 36:456-465.

Wright, S. 1943. Isolation by distance. Genetics 28:114-138.

Yaber, M. C., and K. N. Rabenold. 2002. Effect of sociality on short-distance, female-biased dispersal in tropical wrens. J. Anim. Ecol. 71:1042-1055.

Corresponding Editor: F. Bonhomme

APPENDIX

Symbols used in the model. ${ }^{(1)}$ Symbols with subscript $m$ or $f$ designate a male or a female trait, respectively, whereas symbols without subscript designate juvenile traits.

$g \quad$ Number of groups per population

$d_{1} \quad$ Dispersal rate among groups within populations ${ }^{(1)}$

$d_{2} \quad$ Dispersal rate among populations $\mathrm{s}^{(1)}$

$\gamma \quad$ Coancestry among two individuals ${ }^{(1)}$ from the same group

$\varphi \quad$ Coancestry among two individuals ${ }^{(1)}$ from different groups within the same population

$\theta=\frac{\gamma}{g}+\frac{g-1}{g} \varphi$

$\alpha$

$x$

$z$

$\sigma_{w}^{2}$
$\sigma_{b}^{2}$

$\sigma_{a}^{2}=\theta-\alpha$

$\sigma_{t}^{2}=1-\alpha$

$\sigma_{g}^{2}=\gamma-\varphi$

$\sigma_{c}^{2}=\sigma_{b}^{2}-\frac{g-1}{g} \sigma_{g}^{2}$

$\sigma_{s}^{2}=\sigma_{a}^{2}-\frac{1}{g} \sigma_{g}^{2}=\varphi-\alpha$

$F_{\mathrm{ST}}=\frac{\sigma_{a}^{2}}{\sigma_{t}^{2}}=\frac{\theta-\alpha}{1-\alpha}$

$F_{\mathrm{ST}}^{*}=\frac{\sigma_{s}^{2}}{\sigma_{t}^{2}}=\frac{\varphi-\alpha}{1-\alpha}$

$F_{\mathrm{GT}}^{*}=\frac{\sigma_{g}^{2}+\sigma_{s}^{2}}{\sigma_{t}^{2}}=\frac{\gamma-\alpha}{1-\alpha}$
Coancestry among two individuals ${ }^{(1)}$ from the same population

Coancestry among individuals from different populations

Probability that two individuals ${ }^{(1)}$ sampled within a population stem from different populations

Probability that two individuals ${ }^{(1)}$ sampled within a group stem from different groups from the same population

Genetic variance within individuals

Genetic variance among individuals ${ }^{(1)}$ within populations (nonhierarchical settings)

Genetic variance among populations ${ }^{(1)}$ (nonhierarchical settings)

Total genetic variance in the system

Genetic variance among groups ${ }^{(1)}$ within populations (hierarchical settings)

Genetic variance among individuals ${ }^{(1)}$ within groups (hierarchical settings)

Genetic variance among populations ${ }^{(1)}$ (hierarchical settings)

Proportion of total variance due to differentiation among populations (nonhierarchical settings)

Proportion of variance due to differentiation among populations (hierarchical settings)

Proportion of variance due to differentiation among groups within total (hierarchical settings) 